\title{
RETRACTED ARTICLE: Breaking the Chains of Food Apartheid: Using Youth Participatory Action Research to Spark a Food Revolution in East Oakland
}

\author{
A. A. Akom • Aekta Shah • Aaron Nakai
}

Published online: 17 December 2014

(c) Springer Science+Business Media New York 2015

The Journal Editor has retracted the article "Breaking the Chains of Food Apartheid: Youth Participatory Action Research to Spark a Food Revolution in East Oakland" by A. A. Akom, Aekta Shah, and Aaron Nakai published online in Race and Social Problems (DOI:10.1007/s12552014-9137-y) as it contains portions of text that have been duplicated from the article "Fried Chicken and Fresh Apples: Racial Segregation as a Fundamental Cause of Fast Food Density in Black Neighborhoods" by Naa Oyo A. Kwate published in Health \& Place 2008, 14(1) pages 32-44. We apologize to the readers that this was not detected during the submission and review process.

A. A. Akom - A. Nakai

San Francisco State University, San Francisco, CA, USA

e-mail: akom@sfsu.edu

A. Nakai

e-mail: anakai@iseeed.org

A. A. Akom - A. Shah - A. Nakai

ISEEED, Oakland, CA, USA

A. Shah $(\square)$

Stanford University, Palo Alto, CA, USA

e-mail: aes252@mail.harvard.edu 